\title{
An analysis of abnormalities in the B cell receptor repertoire in patients with systemic sclerosis using high-throughput sequencing
}

\author{
Xiaodong Shi ${ }^{\text {Equal first author, } 1}$, Tihong Shao ${ }^{\text {Equal first author, } 2}$, Feifei Huo ${ }^{3}$, Chenqing Zheng ${ }^{4}$, Wanyu Li ${ }^{\text {Corresp., } 5}$, Zhenyu Jiang \\ Corresp. 1 \\ ${ }^{1}$ Rheumatology, First hospital of Jilin university, Changchun, the People's Republic of China \\ 2 Rheumatology, The First Affiliated Hospital of Anhui Medical University, Hefei, the People's Republic of China \\ 3 Intensive Care Unit, First hospital of Jilin university, Changchun, the People's Republic of China \\ ${ }^{4}$ Shenzhen RealOmics ( Biotech ) Co.Ltd, Shenzhen, the People's Republic of China \\ 5 Hepatology, First hospital of Jilin university, Changchun, the People's Republic of China \\ Corresponding Authors: Wanyu Li, Zhenyu Jiang \\ Email address: 82232559@qq.com, 328430366@qq.com
}

Systemic sclerosis is a chronic multisystem autoimmune disease that is associated with polyclonal B cell hyperreactivity. The CDR3 of BCRs is the major site of antigen recognition. Therefore, we analyzed the BCR repertoire of patients with SSc.The BCR repertoires in 12 subjects including 8 SSc patients and 4 healthy controls were characterized by high-throughput sequencing, and bioinformatics analysis were studied.The average CDR3 length in the SSc group was significantly shorter. The SSC patient displayed more diverse BCR. Moreover, SSc patients with mild skin sclerosis, antiScl70, interstitial lung disease or female sex were more diversified. B cells from the SSC patients showed a differential V and J gene usage. SSc patients had distinct BCR repertoires.These findings reflected the differences of BCR repertoires between SSC patients and controls. The higher-usage genes for the BCR sequence might be potential biomarkers of B cell-targeted therapies or diagnosis for SSC. 
1 An analysis of abnormalities in the $B$ cell receptor repertoire in patients with

2

3

4

5

6

9

\section{systemic sclerosis using high-throughput sequencing}

Xiaodong Shi ${ }^{1}$, Tihong Shao ${ }^{2}$, Feifei Huo ${ }^{3}$, Chenqing Zheng ${ }^{4}$, Wanyu $\mathrm{Li}^{5}$, Zhenyu Jiang ${ }^{1}$

${ }^{\mathbf{1}}$ Rheumatology, First hospital of Jilin university, Changchun, the People's Republic of China

${ }^{2}$ Rheumatology, The First Affiliated Hospital of Anhui Medical University, Hefei, the People's Republic of China

${ }^{\mathbf{3}}$ Intensive Care Unit, First hospital of Jilin university, Changchun, the People's Republic of China

${ }^{4}$ Shenzhen RealOmics ( Biotech ) Co.Ltd, Shenzhen, China

${ }^{\mathbf{5}}$ Hepatology, First hospital of Jilin university, Changchun, the People's Republic of China

These authors (Xiaodong Shi and Tihong Shao) contributed equally to this work.

Corresponding Authors:

Zhenyu Jiang and Wanyu Li

Xinmin street, Changchun, Jilin, 130021, China

Email address:328430366@qq.com ( Zhenyu Jiang )or 82232559@qq.com ( Wanyu Li)

(1)




\section{Abstract}

Systemic sclerosis is a chronic multisystem autoimmune disease that is associated with polyclonal B cell hyperreactivity. The

CDR3 of BCRs is the major site of antigen recognition. Therefore, we analyzed the BCR repertoire of patients with SSc.The BCR repertoires in 12 subjects including $8 \mathrm{SSc}$ patients and 4 healthy controls were characterized by high-throughput sequencing, and bioinformatics analysis were studied.The average CDR3 length in the SSc group was significantly shorter. The SSc patient displayed a more diverse BCR. Moreover, SSc patients with mild skin sclerosis, anti-Scl70, interstitial lung disease or female sex were more diversified. B cells from the SSc patients showed a differential V and J gene usage. SSc patients had distinct BCR repertoires. These findings reflected the differences in BCR repertoires between SSc patients and controls. The higher-usage genes for the BCR sequence might be potential biomarkers of B cell-targeted therapies or diagnosis for SSc.

\section{Introduction}

Systemic sclerosis ( $\mathrm{SSc}$ ) is a chronic multisystem disease characterized by extensive fibrosis, autoantibody production, and microangiopathy. Fibrosis involves the skin and internal organs, including the lung, gastrointestinal tract, and heart. However, the pathogenesis of SSc is not completely understood (Sakkas et al. 2006). Recently, B cells have been highlighted as exerting important regulatory effects independent of their antibody-producing function. B cells promote fibrosis by producing autoantibodies, cytokines, and some mediators, and their infiltration in SSc lesions varies (Hussein et al. 2005; Lafyatis et al. 2007). B cells have also been reported to be involved in the pathogenic mechanism of the disease. B cells are hyperactive in patients with SSc in the presence of hyper- $\gamma$-globulinemia, and increased levels of autoantibodies, free immunoglobulin light chains. (Lanteri et al. 2014). In the B cell repertoire of patients with early SSc, 54\% of the B cells overexpress the stimulatory receptor CD19, whereas $28 \%$ overexpress this receptor in patients with a chronic disease (Mavropoulos et al. 2016). The hyperactivity of B cells in SSc patients results in the overexpression of autoantibodies. Some autoantibodies induce a proinflammatory and/or fibrotic response in patients with SSc (Fineschi et al. 2008; Walker et al. 2007). B cell depletion has been induced in patients with SSc through treatment with an anti-CD20 monoclonal antibody (Jordan et al.2015), which not only ameliorated skin fibrosis but also improved/stabilized lung function (Giuggioli et al. 2015; Mahler et al. 2015; Villalta et al. 2012). An in-depth understanding of B cell genetics and biology will provide more strategies and methods for B cell-targeted therapy.

B cells are selectively activated following the specific recognition of antigens by variable regions of surface $\mathrm{B}$ cell receptors (BCRs). B cell genes encode the variable regions of antibody heavy and light chain proteins. Further mutation is caused by recombination, junctional diversity, and somatic mutations, resulting in as many as $10^{11}$ unique antibody molecules. Three complementary determinant regions (CDR3s) are present in the variable region of the heavy and light chains that are highly susceptible to somatic hypermutations and encode the amino acid loops of the antigen-binding site. Within CDR3s, the CDR3 region of the heavy chain $(\mathrm{VH})$ plays the main role in determining the specificity and diversity of antibodies and antigens bound (Giuggioli et al. 2015; Glanville et al. 2009).

The diversity of BCRs in healthy human peripheral blood is estimated to be approximately $3 \times 10^{9}$, making an analysis of the entire repertoire more difficult(Xu \& Davis 2000). Next-generation sequencing (NGS) technology not only enables largescale DNA sequencing but also enables an in-depth analysis of the BCR repertoire in the circulatory system (Jung et al. 2006), thereby facilitating an in-depth study of the diversity and selection mechanism of the BCR repertoire (Georgiou et al. 2014). 
80

In the present study, we investigate the BCR repertoire in patients with SSc. The CDRs of BCRs were detected using highthroughput sequencing (HTS) to explore the differences in the BCR repertoire between the SSc and healthy control groups. The large-scale sequencing of this BCR repertoire may improve our understanding of the immune system in patients with SSc. In addition, a deeper understanding of the BCR repertoire in patients SSc will help us understand the mechanisms of B cell hyperactivity and tolerance imbalance in patients with SSc, which we hope will provide a theoretical basis for the development of more effective targeted therapies in the future.

\section{Materials and Methods}

\section{Subjects}

In this study, 8 patients with SSc conforming to the criteria of the American College of Rheumatology were included (Calis \& Rosenberg 2014), and patients with a history of or current use of immunosuppressive therapy were excluded. The 8 patients with SSc (SSc group) and 4 healthy controls (Control group) were treated at the First Hospital of Jilin University, Changchun, China, between January and December 2016 and were enrolled after obtaining informed consent and institutional approval. The patients were diagnosed with diffuse cutaneous SSc based on the criteria reported by LeRoy et al. (1980). Four age-matched healthy subjects comprised the control group. We declare that this study has been approved by the Ethics Committee of the First Hospital of Jilin University (2015-119), and written informed consent was obtained from all participants.

The clinical manifestations and laboratory results were derived from clinical questionnaires. Lung fibrosis was observed using high-resolution computed tomography (HRCT). The modified Rodnan total skin thickness score was used to assess the degree of skin fibrosis (LeRoy et al. 1988). The 8 patients scored 12-30 points before treatment and were divided into two groups (mild and severe) according to a cutoff for the modified Rodnan score of 18 points. Table 1 shows the clinical and laboratory characteristics of each patient.

\section{Samples collection}

Ten milliliter blood samples were collected, and peripheral blood mononuclear cells (PBMCs) from patients and controls were obtained immediately by density-gradient centrifugation over Ficoll (MD Pacific Biotechnology Co., Ltd, Tianjin, China). Total RNA was extracted from PBMCs by TRIZOL reagent (Invitrogen, Carlsbad, CA,USA)according to the manufacturer's instructions. Nanodrop 2000 was used to evaluate the quantity and purity of RNA, which was reversely transcribed into cDNA.

\section{Multiplex PCR amplification}

The human immunoglobulin heavy chain (IGH) sequences were downloaded from the international ImMunoGeneTics information system (IMGT) (http://www.imgt.org/). A relatively conserved region 3 was selected as the predicted forward primer region in the upstream sequence of CDR3. A set of primers corresponding to most V gene families were selected. Similarly, 6 reverse primers consistent with $\mathrm{J}$ gene families were designed. MFEprimer2.0 (Shanghai, China) and Oligo 7.0 (Colorado Springs, CO, USA) were used to analyze the primers and reverse primers for dimers and loop structures. We cooperated with the BGI-Shenzhen Company to regulate primer concentrations and minimize PCR amplification bias using a new bioinformatics method (IMonitor) (Clements et al. 1995; Liu et al. 2016). The sequences of low-quality primers were slightly altered. The final primer sequences are shown in supplementary Table S1.

The complete VDJ rearrangements of the IGH sequences were amplified from 4ug of DNA using the primers listed in the IMGT database. Each sample was amplified by performing multiple PCRs (Qiagen multiplex PCR kit) with the same amount of 
119 DNA using a high-fidelity enzyme. The PCR conditions were $15 \mathrm{~min}$ at $95^{\circ} \mathrm{C}, 25$ cycles at $94^{\circ} \mathrm{C}$ for $15 \mathrm{~s}$ and $60^{\circ} \mathrm{C}$ for $3 \mathrm{~min}$, and

120

121

122

123

124

125

126

127

128

129

130

131

132

133

134

135

136

137

138

139

140

141

142

143

144

145

146

147

148

149

150

151

152

153

154

155

156

157

158

159

160

an incubation at $72^{\circ} \mathrm{C}$ for $10 \mathrm{~min}$. PCR products were purified and primer sequences were removed using AMPure XP beads (Brea, CA, USA). Then, the second round of PCR began, and we added a sequencing index to each sample. The PCR conditions were $98^{\circ} \mathrm{C}$ for $1 \mathrm{~min}, 25$ cycles at $98^{\circ} \mathrm{C}$ for $20 \mathrm{~s}, 65^{\circ} \mathrm{C}$ for 30 sand $72^{\circ} \mathrm{C}$ for $30 \mathrm{~s}$, and a final incubation at $72^{\circ} \mathrm{C}$ for 5 min. Finally, an agarose gel was used to isolate the target library, and QIAquick gel Extraction Kits (Qiagen) were used to isolate and purify the target region.

\section{High-throughput sequencing}

The Illumina HiSeq sequence adapter was connected and libraries were sequenced using the Illumina HiSeq 4000 platform. The library was quantified using an Agilent 2100 bioanalyzer (Agilent DNA 1000 reagent, Santa Clara, California, USA) and real-time quantitative PCR (TaqMan probes, Shenzhen, China).

Data analysis

The quality of the Illumina HiSeq 4000 library was evaluated using a BGI formula. Namely, we filtered adapter reads and lowquality reads in the raw data, and further aligned clean data. The clean data were then compared with the human IGH database and analyzed with MiXCR, which categorized the identical and homologous reads into clonotypes and corrected the PCR and sequencing errors using heuristic multi-layer clustering. The obtained data included V, D, J assignments, clustering, CDR3 length distributions and other results.

\section{Statistical analyses}

Statistical analyses were conducted in R software. The Wilcoxon rank-sum test was used to compare the expression level and expression diversity between the SSC and the control group. A two-sided $\mathrm{P}<0.05$ was considered statistically significant.

\section{Results}

\section{Summary of the sequencing results}

Eight patients with SSc (SSc group: P1-8) and four healthy controls (Control group: H1-4) were enrolled in this study. After filtering, including the removal of adapter sequences, contamination, and low-quality reads, we acquired an average of $8,363,668$ sequencing reads each sample. Table 2 showed the IGH sequence summary. (Table 2)

\section{Distribution of CDR3 lengths}

The important determinant of the diversity of the B cell repertoire is the length of the BCR CDR3 loop. In our study, we evaluated the length distribution of the BCR CDR3 sequence (aa) in the SSc and Control groups. The SSc group had a higher percentage of BCR CDR3 sequences of 14 amino acids (aa) in length than the Control group ( $\mathrm{P}=0.029)$, but a lower percentage of BCR CDR3 sequences of $29(\mathrm{P}=0.039)$ or $37(\mathrm{P}=0.013)$ aa in length (Figure $\mathrm{S} 1)$. The average CDR3 length was significantly shorter in the $\mathrm{SSc}$ group (17.85 $\pm 0.37 \mathrm{aa})$ than in the Control group (18.47 \pm 0.038 aa; $\mathrm{P}=0.038$ ) (Figure $\mathrm{S} 2$ ).

\section{Degree of expansion and frequency distribution of $B$ cell clones}

By aligning and identifying each sequence, we were able to calculate the expression level of each clone. The extent to which each individual clone expands depends on the frequency of the unique CDR3 sequence in a sample. Here, we defined clones with a frequency greater than $0.5 \%$ among the analyzed BCRs as highly expanded clones (HECs), clones with a frequency of 0.05 $0.5 \%$ as high-frequency clones, clones with a frequency of $0.005-0.05 \%$ as medium-frequency clones, clones with a frequency of $0.0005-0.005 \%$ as low-frequency clones, and clones with a frequency of $<0.0005 \%$ as rare clones. In terms of the frequency distribution, most of the repertoires were composed of a small amount of HECs distributed in a left-skewed manner, and most clones were present at a low frequency (Figure S3). In the SSc group, the more highly expanded clones $\left(9.37 \times 10^{-2} \pm 3.51 \times 10^{-2} \%\right)$ accounted for $10 \%$ of the $\mathrm{B}$ cell sequences present among the medium-frequency clones $(0.005-0.05 \%)(\mathrm{P}=0.005)$, and the 
expanded clones $\left(1.31 \times 10^{-2} \pm 1.42 \times 10^{-2} \%\right)$ accounted for $2.1 \%$ of the B cell sequences present among the high-frequency clones $(0.05-0.5 \%)(\mathrm{P}=0.013)$. However, lower expression levels were observed among the rare clones $\left(0.874 \pm 3.32 \times 10^{-2 \%}\right)(\mathrm{P}=0.019)$ in the SSc group than in the Control group (TableS2).

\section{Comparison of the BCR repertoire diversity between groups}

We used the Shannon entropy index, which summarizes the frequency of every clonotype, to quantify the BCR repertoire diversity of the SSc group, Control group and subgroups with different features. An analysis of the repertoires of patients with $\mathrm{SSc}$ revealed a very high $\mathrm{BCR}$ diversity that was higher than the Control group $(\mathrm{P}=0.004)$. Moreover, the entire $\mathrm{BCR}$ repertoire of the SSc group had a much more diversified clonotype composition than the Control group, particularly in the subgroups with a mild degree of skin sclerosis, anti-Scl70 antibodies, or interstitial lung disease (ILD). Furthermore, the Shannon entropy of the Control group and female and male patients in the SSc group was analyzed; the BCR diversity of female patients was higher than the Control group. Although the $\mathrm{P}$ value was not statistically significant, a greater diversity was observed in male patients than in female patients. The sample size of the male group should be increased in subsequent studies (Figure 1).

\section{Distribution of similar CDR3 sequences in the SSc and Control groups}

The similarity map of the samples was obtained by calculating the distance between each pair of samples as the Jaccard distance $=1-$ Jaccard index and constructing the neighbor-joining tree (Figure 2). According to the map, the subjects were divided into three groups: female patients with SSc (P1-6), male patients with SSc (P7-P8) and healthy controls (H1-4). An obvious difference was detected between the patients and healthy controls, which were well-separated; however, among the patients, the similarities between P7 and P8 were also different from the other patients. (The sequences in male patients with SSc were also different from those in female patients with SSc.)

\section{Comparison of IGHV and IGHJ repertoires between the SSc and Control groups}

The usage features of the $\mathrm{V}$ and $\mathrm{J}$ gene segments were analyzed for the different clone levels, as shown by the histograms and heat maps, respectively (Figures 3 and 4), to analyze whether disease-specific differences in the IGHV and IGHJ repertoires existed. The expression levels of the respective IGHV and IGHJ repertoires were compared among the groups. For the IGHV gene segments, IGHV3-9 ( $\mathrm{P}=0.04)$ were highly expressed in the $\mathrm{SSc}$ group. For the IGHJ gene segments, IGHJ4 (P=0.02) showed significantly higher usage in the SSc group. Then, the relative usage frequencies of V-J pairs were compared between the SSc and Control groups (Figures 3C and 4, table 3). IGHV1-18-J3,GHV1-8-J2,GHV1-8-J4, IGHV3-53-J4,IGHV3-9J3,IGHV3-9-J4,IGHV3-9-J5, IGHV4-61-J4, SSc group is higher than Control (Figures 3C and 4); and in IGHV2-5-J6,IGHV270-J5, IGHV3-20-J6, IGHV3-23-J6, IGHV3-7-J5, IGHV4-4-J6, SSc group is lower than Control.(Figure 4,table3)

\section{Discussion}

B cells are hyperactivated and produce many autoantibodies in patients with SSc, some of which can lead to collagen production and vasoconstriction (Zhang et al. 2015). NGS of the B cell receptor is helping researchers understand and identify important questions in the field of B cell repertoire development and differences in the B cell subsets in healthy and diseased states. B cells play important roles in various autoimmune diseases, particularly in the occurrence and development of SSc, affecting the immune response of patients to antigens (Sakkas \& Bogdanos 2016; Schanz et al. 2014). The most variable region of the BCR sequence is the CDR3 region, which directly determines the specificity of antigen binding to the BCR (BashfordRogers et al. 2018). HTS allows a detailed investigation of the BCR repertoire. To date, the composition of and variations in the BCR VH CDR3 repertoire of patients with SSc have not been reported. In the present study, we employed a novel NGS method 
202

203

204

205

206

207

208

209

210

211

212

213

214

215

216

217

218

219

220

221

222

223

224

225

226

227

228

229

230

231

232

233

234

235

236

237

238

239

240

241

to compare and analyze the repertoire of the BCR IGHV CDR3 region in a group of patients with SSc ( $\mathrm{n}=8)$ and a group of healthy controls $(n=4)$.

We analyzed the distributions of CDR3 lengths among an average of 8,363,668 filtered sequencing reads per sample, which provided extensive information on the BCR repertoire in the SSc and Control groups. For example, we identified the most frequently observed length. Variable rearrangements cause different CDR3 lengths, the characteristics of BCR clonality are determined by measuring the lengths of CDR3 subsets. However, in our study, the distribution of BCR CDR3 lengths showed a higher percentage of BCR CDR3 sequences of 14 aa in length in the SSc group than in the Control group (P=0.029), but a lower percentage of $\mathrm{BCR}$ CDR3 sequences of 29 aa $(\mathrm{P}=0.039)$ or 37 aa $(\mathrm{P}=0.013)$ in length in the $\mathrm{SSc}$ group. In addition, the average CDR3 length in the SSc group (17.85 \pm 0.37 aa) was significantly shorter than in the Control group (18.47 \pm 0.038 aa; $\mathrm{P}=0.038)$. Interestingly, the length of the BCR CDR3 is also significantly shorter in patients with systemic lupus erythematosus (SLE) than in the controls(Robins 2013). However, this effect may be due to the continuous increase in the proportion of plasmablasts in patients with SLE, since the CDR3 length of BCRs on naïve B cells is often longer than on B cells with antigen experience(Liu et al. 2017). Moreover, B cell clones from patients with rheumatoid arthritis (RA) are enriched for longer heavy chain CDR3 lengths (Doorenspleet et al. 2014; Galson et al. 2015; Samuels et al. 2005). In the present study, we mainly observed the B cell repertoire of patients with SSc and healthy controls, and found that the number of different antibody types and B cell subsets were not well differentiated. Based on our results, patients with SSc have CDR3 regions with shorter lengths in the overall repertoire. Thus, the repertoire of different B cell subsets in patients with SSc requires further research.

The Shannon entropy index and the distribution of HECs differed between the two groups. Most individual B cell clones occurred at very low frequencies, suggesting that these clones have not yet expanded. However, the expansion of clones was observed in both groups; among medium-frequency clones, a greater degree of expansion was observed in the SSc group than in Control group (0.005-0.05\%) $(\mathrm{P}=0.005)$. One possible explanation for this difference is that the existence of autoantigens induces the development of new autoreactive clones.

The diversity analysis revealed that the repertoires of patients with SSc exhibited a much higher BCR diversity than the Control group $(\mathrm{P}=0.004)$. Moreover, the clonotype composition of the $\mathrm{BCR}$ repertoire was much more diversified in patients with SSc presenting a mild degree of skin sclerosis, anti-Scl70 antibodies, ILD or the female sex than in the controls. As shown in the study by Forestier et al. (Forestier et al. 2018), B cells undergo clonal expansion in response to chronic stimulation, which is potentially caused by autoantigens or pathogens. In addition, variations in IGHV genes are associated with disease susceptibility. Both patients with SSc and tight-skin mice (the genetic model of SSc) display intrinsic B cell abnormalities that are primarily characterized by chronic B cell activation (Brezinschek et al. 1997; Hasegawa 2010; Saito et al. 2002). Based on our results, the higher BCR diversity might be associated with the occurrence and development of SSc.

Additionally, although the size of males of SSc patients was small, and it was still not statistically comparable with the female and control groups, the diversity of male SSc patients tends to increase according to the data ( Figure 1e).Consistent with this finding, a noticeable difference in the distribution of similar CDR3 sequences was observed between patients and healthy controls by calculating the distance between each pair of samples (Figure 2). Furthermore, among the patients, the similarity of the male patients (P7 and P8) was obviously different from the female patients. Sex hormones may play a role in sex-differences in autoimmune diseases. Most human autoimmune diseases, like systemic lupus erythematosus(SLE), Sjogren's syndrome (SS) etc., have increased incidence and prevalence in females(Lee \& Chiang 2012), a study has shown that examination of the B cell subsets in estrogen-treated groups revealed the reduction in the number of transitional B cells, B cell lymphopoiesis is reduced in both pregnant and in the estrogen-treated population (Bynoe et al. 2000). In our study, the diversity of the BCR repertoire in male patients with SSc is different from female patients, It may be that the B cells subsets, growth and activation states and 
immune response in sex hormones differ. We need to increase the sample size of the male patient to further confirm a different state of the male human antibody repertoire in systemic sclerosis.

BCR repertoires in patients with SSc showed significant changes in IGHV gene usage compared to healthy controls. Our results showed an enrichment of IGHV3-9 and IGHJ4 gene family usage, specificallyIGHV1-18-J3, GHV1-8-J2, GHV1-8-J4, IGHV3-53-J4, IGHV3-9-J3, IGHV3-9-J4, IGHV3-9-J5 and IGHV4-61-J4 usage in patients with SSc was higher than Control, but in IGHV2-5-J6, IGHV2-70-J5, IGHV3-20-J6, IGHV3-23-J6, IGHV3-7-J5, IGHV4-4-J6, the usage was lower than Control. The usage of these pairs was significantly different between the SSc and Control groups (Figures 3, Figure 4 and table 3). These enrichments, particularly the usage of IGHV1 and IGHV4 and the IGHV3, IGHJ4, and IGHJ6 combinations, have also been observed in studies of multiple autoimmune diseases (Arbuckle et al. 2003; Odendahl et al. 2000; Shi et al. 2016; Tipton et al. 2015). Furthermore, our study has shown that the usage of IGHV2-5-J6 in SSc was significantly lower than control, which is consistent with de Bourcy's study, IGHV2-5 was used at a lower level in SSc-PAH participants (de Bourcy et al. 2017). It has been proved that B cells changes of systemic sclerosis is related to the balance in naive and memory B cell, and that has been shown, an imbalance in naive/switched and unswitched memory B cells that may explain the B cell repertoire abnormality observed in our study (Simon et al. 2016). In the study, the higher-usage genes also provide additional information for the future study of effective B cell-targeted therapy or prognostic and/or diagnostic biomarkers for SSc.

In conclusion, the analysis of the BCR immune repertoires of patients with SSc using this meaningful method has an important application value for studying the prognosis and evaluating the clinical responses to treatment in the future. Although this study has some limitations due to the small sample size, future investigations aimed at improving our understanding of the role of the BCR repertoire in immune responses, autoimmunity and autoreactivity are anticipated as the cost of HTS decreases: first to analyze the repertoires of different B cell subsets in patients with SSc, and second to further identify the features of the $\mathrm{BCR}$ repertoire and the functions of specific BCR genes in different patients with SSc.

\title{
Acknowledgements
}

\author{
Not applicable.
}

\section{References}

Preliminary criteria for the classification of systemic sclerosis (scleroderma).1980. Subcommittee for scleroderma criteria of the American Rheumatism Association Diagnostic and Therapeutic Criteria Committee. Arthritis Rheum 23:581-590.

Arbuckle MR, McClain MT, Rubertone MV, Scofield RH, Dennis GJ, James JA, and Harley JB. 2003. Development of autoantibodies before the clinical onset of systemic lupus erythematosus. $N$ Engl J Med 349:1526-1533.

Bashford-Rogers RJM, Smith KGC, and Thomas DC. 2018. Antibody repertoire analysis in polygenic autoimmune diseases. Immunology 155:3-17.

Brezinschek HP, Foster SJ, Brezinschek RI, Dorner T, Domiati-Saad R, and Lipsky PE. 1997. Analysis of the human VH gene repertoire. Differential effects of selection and somatic hypermutation on human peripheral CD5(+)/IgM+ and CD5(-)/IgM+ B cells. J Clin Invest 99:2488-2501.

Bynoe MS, Grimaldi CM, and Diamond B. 2000. Estrogen up-regulates Bcl-2 and blocks tolerance induction of naive B cells. Proc Natl Acad Sci U S A 97:2703-2708. 10.1073/pnas.040577497 040577497 
Calis JJ, and Rosenberg BR. 2014. Characterizing immune repertoires by high throughput sequencing: strategies and applications. Trends Immunol 35:581-590. 10.1016/j.it.2014.09.004

Clements P, Lachenbruch P, Siebold J, White B, Weiner S, Martin R, Weinstein A, Weisman M, Mayes M, Collier D. 1995. Inter and intraobserver variability of total skin thickness score (modified Rodnan TSS) in systemic sclerosis. J Rheumatol 22:1281-1285.

de Bourcy CFA, Dekker CL, Davis MM, Nicolls MR, and Quake SR. 2017. Dynamics of the human antibody repertoire after B cell depletion in systemic sclerosis. Sci Immunol 2.

10.1126/sciimmunol.aan82892/15/eaan8289

Doorenspleet ME, Klarenbeek PL, de Hair MJ, van Schaik BD, Esveldt RE, van Kampen AH, Gerlag DM, Musters A, Baas F, Tak PP, and de Vries N. 2014. Rheumatoid arthritis synovial tissue harbours dominant B-cell and plasma-cell clones associated with autoreactivity. Ann Rheum Dis 73:756-762. 10.1136/annrheumdis2012-202861

Fineschi S, Goffin L, Rezzonico R, Cozzi F, Dayer JM, Meroni PL, and Chizzolini C. 2008. Antifibroblast antibodies in systemic sclerosis induce fibroblasts to produce profibrotic chemokines, with partial exploitation of toll-like receptor 4. Arthritis Rheum 58:3913-3923. 10.1002/art.24049

Forestier A, Guerrier T, Jouvray M, Giovannelli J, Lefevre G, Sobanski V, Hauspie C, Hachulla E, Hatron PY, Zephir H, Vermersch P, Labalette M, Launay D, and Dubucquoi S. 2018. Altered B lymphocyte homeostasis and functions in systemic sclerosis. Autoimmun Rev 17:244-255. 10.1016/j.autrev.2017.10.015

Galson JD, Truck J, Fowler A, Clutterbuck EA, Munz M, Cerundolo V, Reinhard C, van der Most R, Pollard AJ, Lunter G, and Kelly DF. 2015. Analysis of B Cell Repertoire Dynamics Following Hepatitis B Vaccination in Humans, and Enrichment of Vaccine-specific Antibody Sequences. EBioMedicine 2:2070-2079. 10.1016/j.ebiom.2015.11.034

Georgiou G, Ippolito GC, Beausang J, Busse CE, Wardemann H, and Quake SR. 2014. The promise and challenge of high-throughput sequencing of the antibody repertoire. Nat Biotechnol 32:158-168. 10.1038/nbt.2782

Giuggioli D, Lumetti F, Colaci M, Fallahi P, Antonelli A, and Ferri C. 2015. Rituximab in the treatment of patients with systemic sclerosis. Our experience and review of the literature. Autoimmun Rev 14:1072-1078.

Glanville J, Zhai W, Berka J, Telman D, Huerta G, Mehta GR, Ni I, Mei L, Sundar PD, Day GM, Cox D, Rajpal A, and Pons J. 2009. Precise determination of the diversity of a combinatorial antibody library gives insight into the human immunoglobulin repertoire. Proc Natl Acad Sci U S A 106:20216-20221.

Hasegawa M. 2010. B lymphocytes: shedding new light on the pathogenesis of systemic sclerosis. $J$ Dermatol 37:310. 10.1111/j.1346-8138.2009.00763.x

Hussein MR, Hassan HI, Hofny ER, Elkholy M, Fatehy NA, Abd Elmoniem AE, Ezz El-Din AM, Afifi OA, and Rashed HG. 2005. Alterations of mononuclear inflammatory cells, CD4/CD8+ T cells, interleukin 1beta, and tumour necrosis factor alpha in the bronchoalveolar lavage fluid, peripheral blood, and skin of patients with systemic sclerosis. J Clin Pathol 58:178-184. 10.1136/jcp.2004.019224

Jordan S, Distler JH, Maurer B, Huscher D, van Laar JM, Allanore Y, Distler O, and group ERs. 2015. Effects and safety of rituximab in systemic sclerosis: an analysis from the European Scleroderma Trial and Research (EUSTAR) group. Ann Rheum Dis 74:1188-1194. 10.1136/annrheumdis-2013-204522

Jung D, Giallourakis C, Mostoslavsky R, and Alt FW. 2006. Mechanism and control of V(D)J recombination at the immunoglobulin heavy chain locus. Annu Rev Immunol 24:541-570.

Lafyatis R, O'Hara C, Feghali-Bostwick CA, and Matteson E. 2007. B cell infiltration in systemic sclerosis- 
associated interstitial lung disease. Arthritis Rheum 56:3167-3168. 10.1002/art.22847

Lanteri A, Sobanski V, Langlois C, Lefevre G, Hauspie C, Sanges S, Lambert M, Morell-Dubois S, Hatron PY, Hachulla E, Launay D, and Dubucquoi S. 2014. Serum free light chains of immunoglobulins as biomarkers for systemic sclerosis characteristics, activity and severity. Autoimmun Rev 13:974-980.

Lee TP, and Chiang BL. 2012. Sex differences in spontaneous versus induced animal models of autoimmunity. Autoimmun Rev 11:A422-429. 10.1016/j.autrev.2011.11.020 S1568-9972(11)00294-1

LeRoy EC, Black C, Fleischmajer R, Jablonska S, Krieg T, Medsger TA, Jr., Rowell N, and Wollheim F. 1988. Scleroderma (systemic sclerosis): classification, subsets and pathogenesis. J Rheumatol 15:202-205.

Liu S, Hou XL, Sui WG, Lu QJ, Hu YL, and Dai Y. 2017. Direct measurement of B-cell receptor repertoire's composition and variation in systemic lupus erythematosus. Genes Immun 18:22-27. 10.1038/gene.2016.45

Liu X, Zhang W, Zeng X, Zhang R, Du Y, Hong X, Cao H, Su Z, Wang C, Wu J, Nie C, Xu X, and Kristiansen K. 2016. Systematic Comparative Evaluation of Methods for Investigating the TCRbeta Repertoire. PLoS One 11:e152464. 10.1371/journal.pone.0152464

Mahler M, Fritzler MJ, and Satoh M. 2015. Autoantibodies to the mitochondrial RNA processing (MRP) complex also known as Th/To autoantigen. Autoimmun Rev 14:254-257.

Matsushita T, Hasegawa M, Yanaba K, Kodera M, Takehara K, and Sato S. 2006. Elevated serum BAFF levels in patients with systemic sclerosis: enhanced BAFF signaling in systemic sclerosis B lymphocytes. Arthritis Rheum 54:192-201. 10.1002/art.21526

Mavropoulos A, Simopoulou T, Varna A, Liaskos C, Katsiari CG, Bogdanos DP, and Sakkas LI. 2016. Breg Cells Are Numerically Decreased and Functionally Impaired in Patients With Systemic Sclerosis. Arthritis Rheumatol 68:494-504. 10.1002/art.39437

Odendahl M, Jacobi A, Hansen A, Feist E, Hiepe F, Burmester GR, Lipsky PE, Radbruch A, and Dorner T. 2000. Disturbed peripheral B lymphocyte homeostasis in systemic lupus erythematosus. J Immunol 165:59705979.

Robins H. 2013. Immunosequencing: applications of immune repertoire deep sequencing. Curr Opin Immunol 25:646-652. 10.1016/j.coi.2013.09.017

Saito E, Fujimoto M, Hasegawa M, Komura K, Hamaguchi Y, Kaburagi Y, Nagaoka T, Takehara K, Tedder TF, and Sato S. 2002. CD19-dependent B lymphocyte signaling thresholds influence skin fibrosis and autoimmunity in the tight-skin mouse. J Clin Invest 109:1453-1462. 10.1172/JCI15078

Sakkas LI, and Bogdanos DP. 2016. Systemic sclerosis: New evidence re-enforces the role of B cells. Autoimmun Rev 15:155-161. 10.1016/j.autrev.2015.10.005

Sakkas LI, Chikanza IC, and Platsoucas CD. 2006. Mechanisms of Disease: the role of immune cells in the pathogenesis of systemic sclerosis. Nat Clin Pract Rheumatol 2:679-685. 10.1038/ncprheum0346

Samuels J, Ng YS, Coupillaud C, Paget D, and Meffre E. 2005. Impaired early B cell tolerance in patients with rheumatoid arthritis. J Exp Med 201:1659-1667. 10.1084/jem.20042321

Schanz M, Liechti T, Zagordi O, Miho E, Reddy ST, Gunthard HF, Trkola A, and Huber M. 2014. High-throughput sequencing of human immunoglobulin variable regions with subtype identification. PLoS One 9:e111726. 10.1371/journal.pone.0111726

Shi B, Yu J, Ma L, Ma Q, Liu C, Sun S, Ma R, and Yao X. 2016. Short-term assessment of BCR repertoires of SLE patients after high dose glucocorticoid therapy with high-throughput sequencing. Springerplus 5:75. 
10.1186/s40064-016-1709-4

Simon D, Balogh P, Bognar A, Kellermayer Z, Engelmann P, Nemeth P, Farkas N, Minier T, Lorand V, Czirjak L, and Berki T. 2016. Reduced non-switched memory B cell subsets cause imbalance in B cell repertoire in systemic sclerosis. Clin Exp Rheumatol 34 Suppl 100:30-36. 9774

Tipton CM, Fucile CF, Darce J, Chida A, Ichikawa T, Gregoretti I, Schieferl S, Hom J, Jenks S, Feldman RJ, Mehr R, Wei C, Lee FE, Cheung WC, Rosenberg AF, and Sanz I. 2015. Diversity, cellular origin and autoreactivity of antibody-secreting cell population expansions in acute systemic lupus erythematosus. Nat Immunol 16:755-765. 10.1038/ni.3175

Villalta D, Imbastaro T, Di Giovanni S, Lauriti C, Gabini M, Turi MC, and Bizzaro N. 2012. Diagnostic accuracy and predictive value of extended autoantibody profile in systemic sclerosis. Autoimmun Rev 12:114-120. 10.1016/j.autrev.2012.07.005

Walker UA, Tyndall A, Czirjak L, Denton C, Farge-Bancel D, Kowal-Bielecka O, Muller-Ladner U, BocelliTyndall C, and Matucci-Cerinic M. 2007. Clinical risk assessment of organ manifestations in systemic sclerosis: a report from the EULAR Scleroderma Trials And Research group database. Ann Rheum Dis 66:754-763. 10.1136/ard.2006.062901

$\mathrm{Xu}$ JL, and Davis MM. 2000. Diversity in the CDR3 region of $\mathrm{V}(\mathrm{H})$ is sufficient for most antibody specificities. Immunity 13:37-45.

Zhang W, Du Y, Su Z, Wang C, Zeng X, Zhang R, Hong X, Nie C, Wu J, Cao H, Xu X, and Liu X. 2015. IMonitor: A Robust Pipeline for TCR and BCR Repertoire Analysis. Genetics 201:459-472. 10.1534/genetics.115.176735 


\section{Figure 1}

Figure 1. The BCR repertoire diversity among SSc patients compared with Control group.

Figure 1. The BCR repertoire diversity among SSc patients compared with Control group. (a) Diversity between the SSc and Control $(p=0.004)$; (b) Shannon entropy among the mild and severe degrees of skin sclerosis patients compared with Control group respectively, ( $p=0.02$ ); (c) Shannon entropy among antinuclear antibody (ANA)-positive and Scl-70positive subgroups compared with Control respectively, $(p=0.03)$; (d) Shannon entropy among the ILD( $p=0.02)$ and Non-ILD patients compared with Control group respectively. (e) Shannon entropy among the female $(p=0.009)$ and male SSc patients compared with Control group. Differences were statistically analyzed for significance by the Wilcoxon rank sum test. 

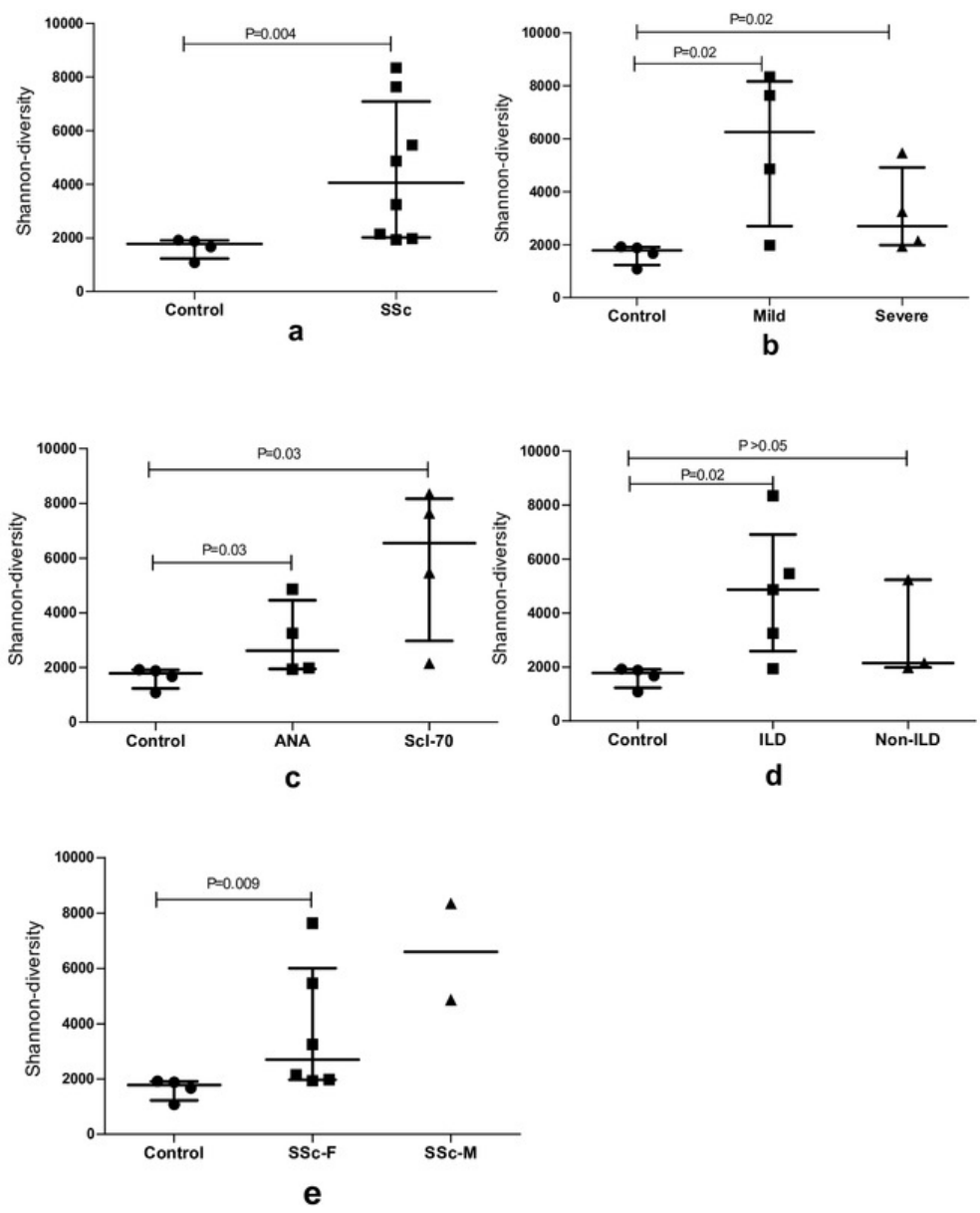


\section{Figure 2}

Figure 2. Similarity distribution of CDR3 sequences in the SSC and Control groups.

Figure 2. Similarity distribution of CDR3 sequences in the SSC and Control groups by calculating the Jaccard distance between each pair of samples. The subjects were divided into three groups according to the figure: female patients with SSC (P1-6), male patients with SSc (P7-P8) and healthy controls (H1-4). 


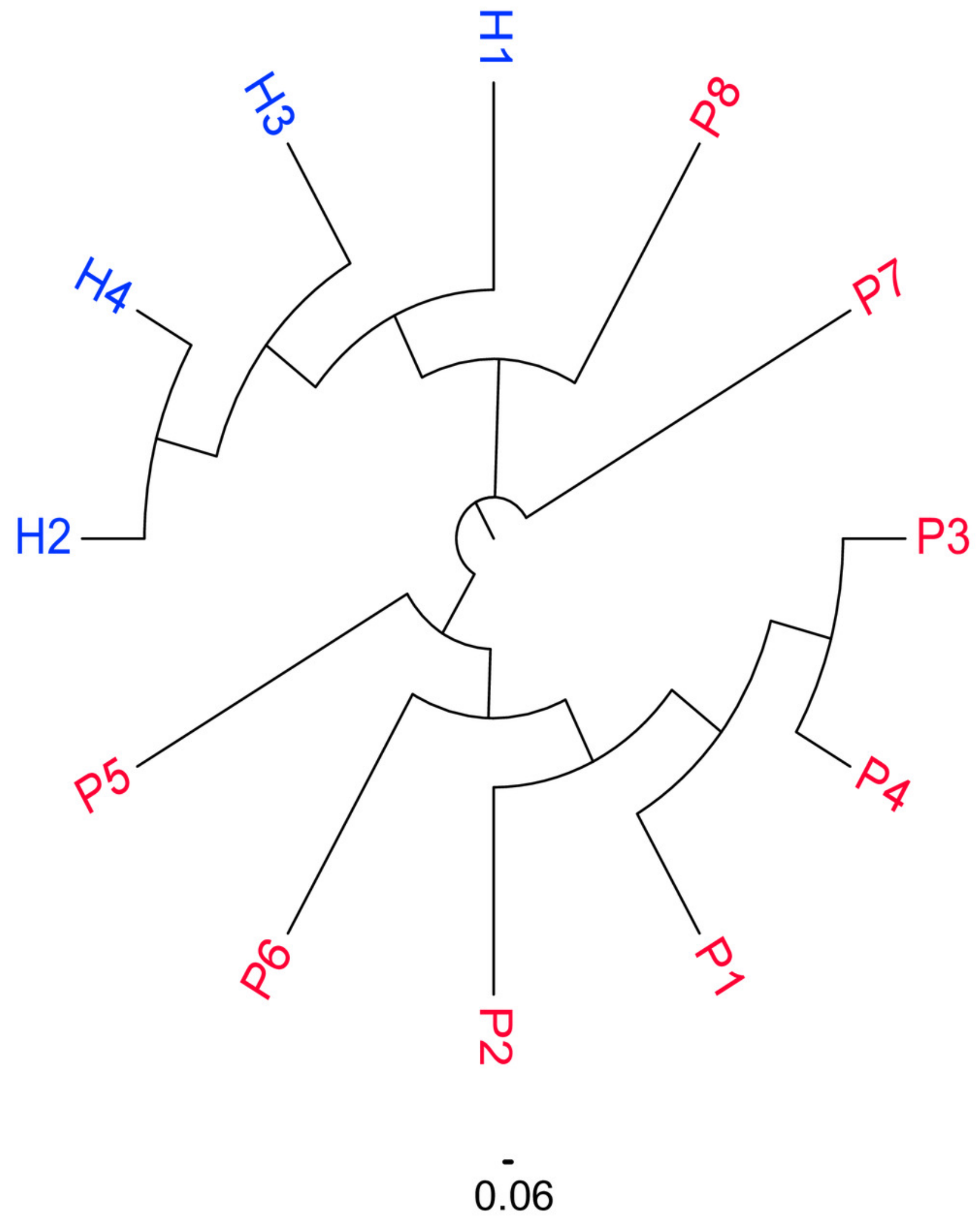




\section{Figure 3}

Figure 3. Relative frequencies of each IGHV gene

Figure 3. Relative frequencies of each IGHV gene (A) [IGH V3-9:P=0.032 ], IGH] gene; (B) [IGHJ4:P=0.028] and IGH V-J; (C) [IGHV3-9-J4:P=0.041; IGHV3-9-J5:P=0.023; IGHV4-4J6:P=0.016; IGHV3-7-J5:P=0.045; IGHV3-23-J6:P=0.016; IGHV3-53-J4:P=0.016;IGHV1-8$J 4: P=0.016]$ fragments in PBMCs from the SSC and Control groups. The bars and error bars indicate the mean frequencies and standard deviations of the results from individual subjects. Differences were statistically analyzed for significance by the Wilcoxon rank sum test $(* \mathrm{P}<0.05)$. 

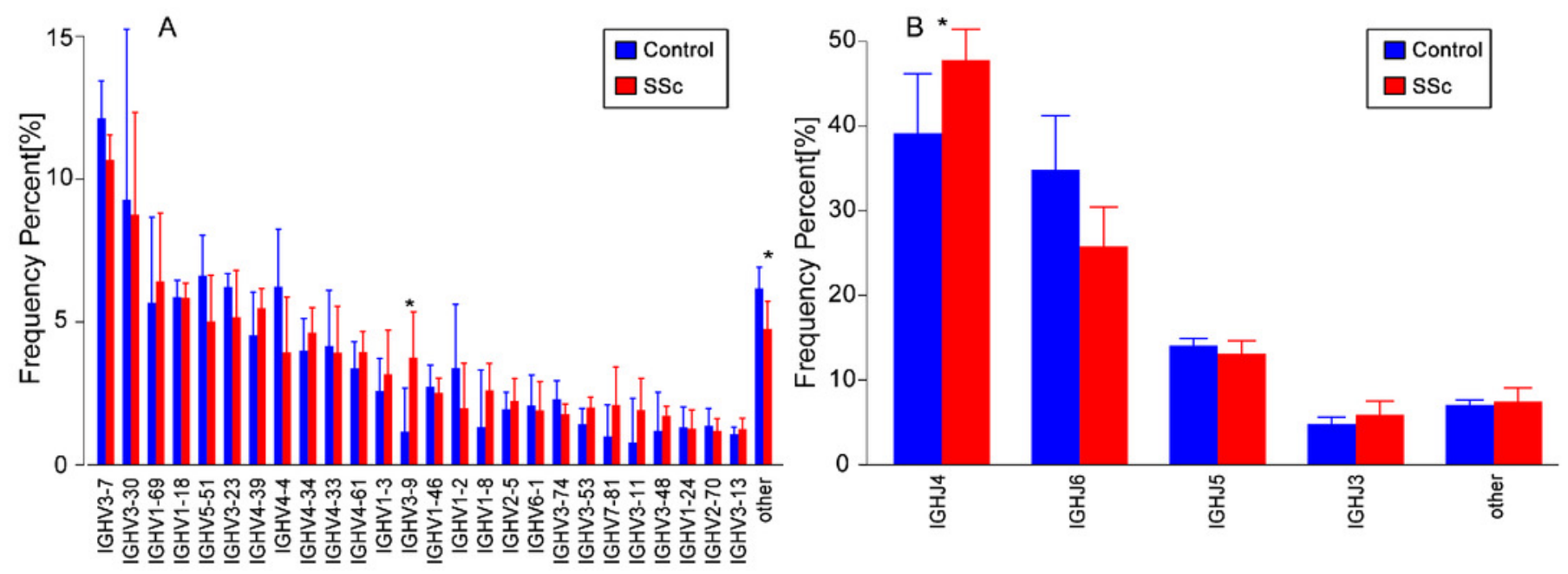

(⿸丆口

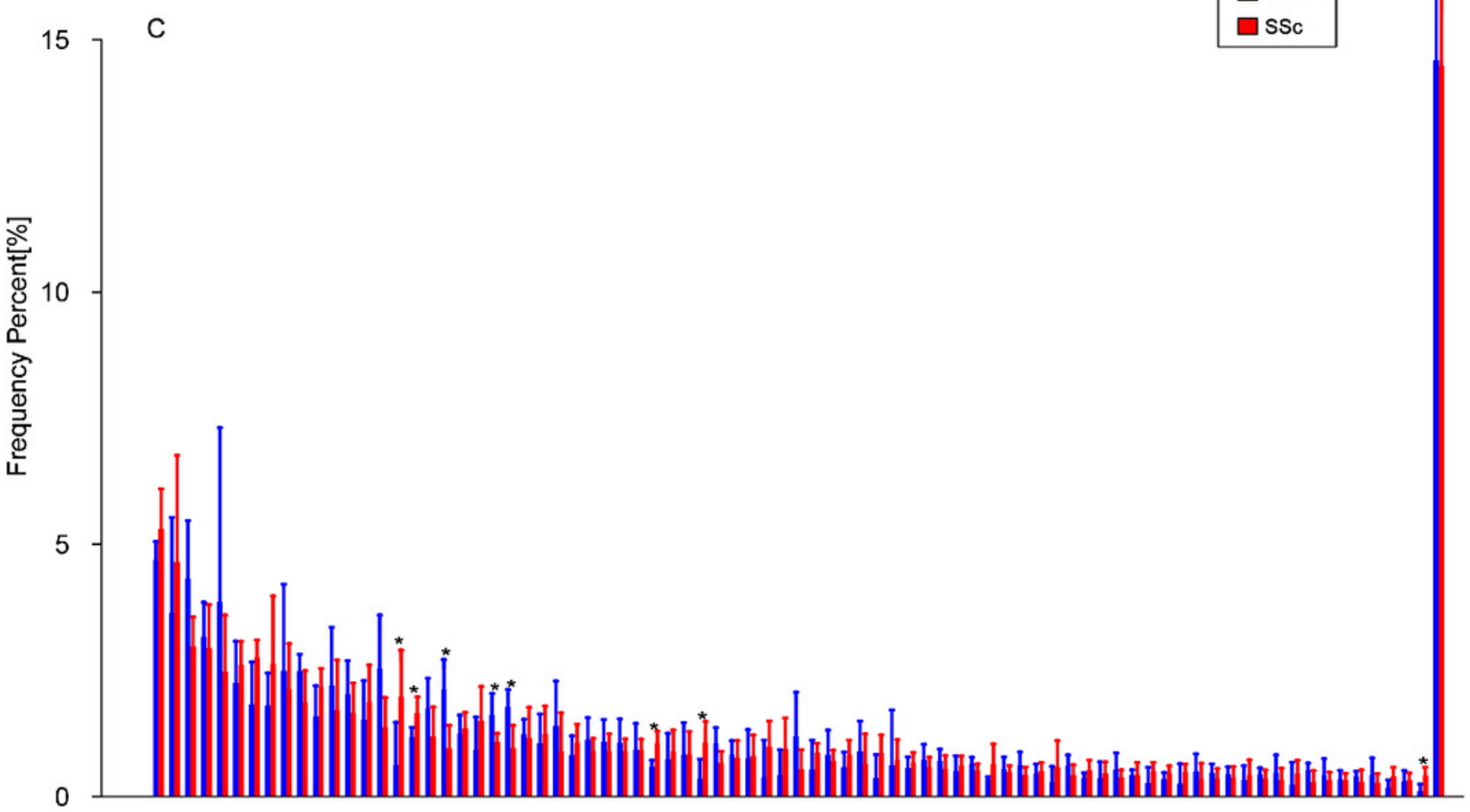

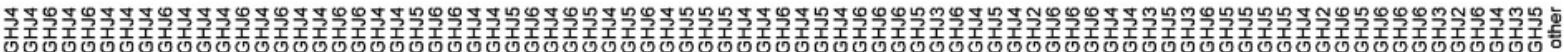
等

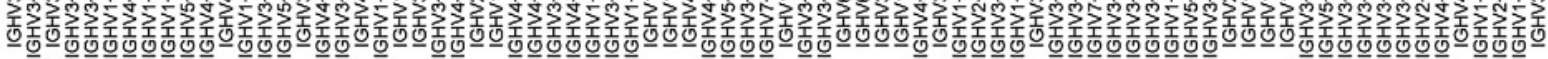




\section{Figure 4}

Figure 4. Comparison of the average V-J gene utilization of the sequenced IGH sequences between the SSc group and Control group.

Figure 4. Comparison of the average V-J gene utilization of the sequenced IGH sequences between the SSc group and Control group. The J gene segments are arranged on the y-axis, and the $\mathrm{V}$ gene segments are arranged on the $\mathrm{x}$-axis. The different colors (red to blue rectangular bands) indicate the different levels of significance. Rectangular bands is the value converted from - $\log 10$ ( $p$ value), "+ " indicates significant difference (SSc group is higher than Control), and " $\square$ " indicates significant difference (Control group is higher than SSc).Differences were statistically analyzed for significance by the Wilcoxon rank sum test $(p<0.05)$. 


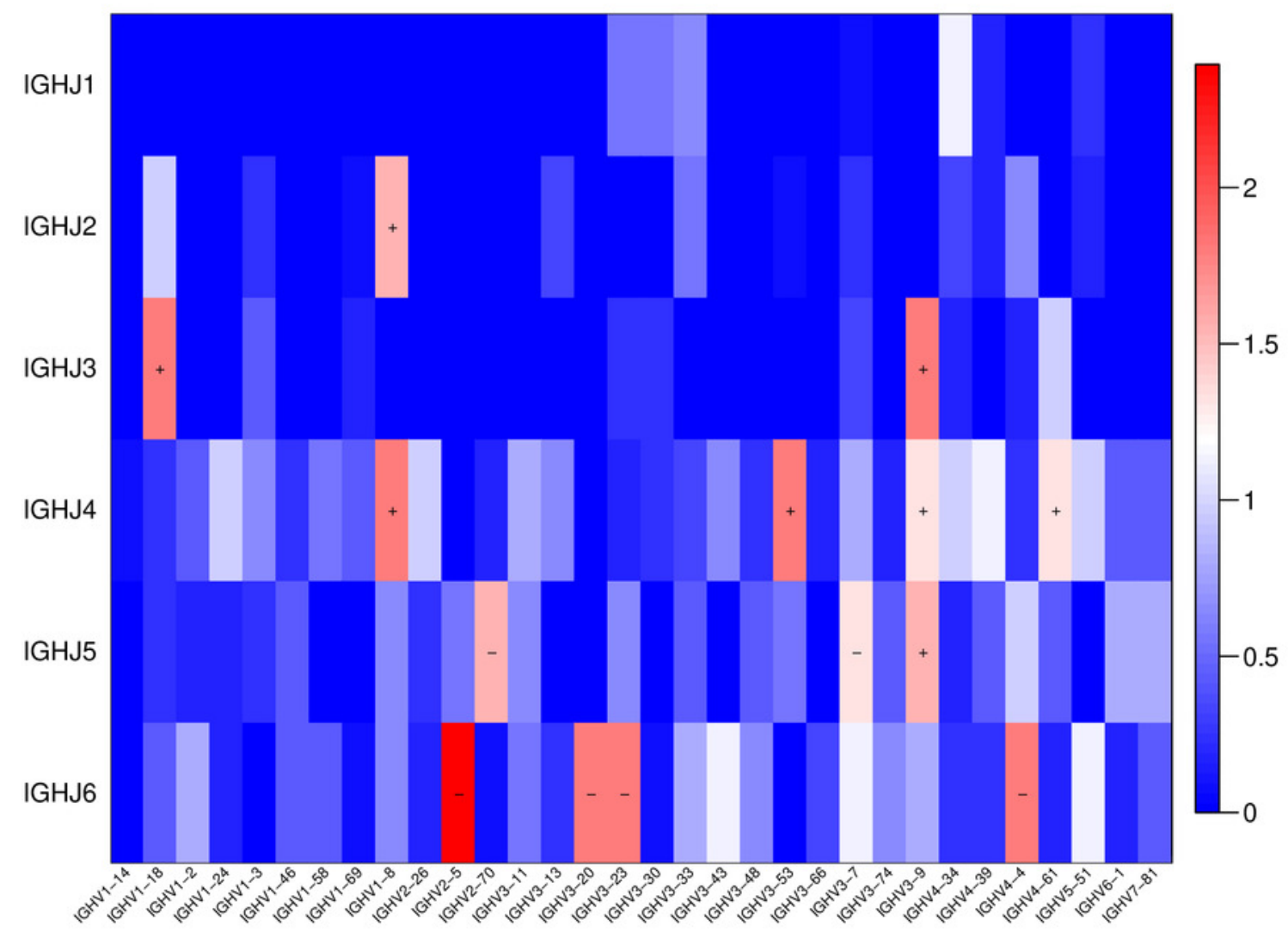




\section{Table $\mathbf{1}$ (on next page)}

Table 1. Clinical characteristics of the study sample.

Table 1. Clinical characteristics of the study sample. 
Table 1. Clinical characteristics of the study sample.

\begin{tabular}{|c|c|c|c|c|c|c|}
\hline ID & Age(years) & Sex & Autoantibodies & $\begin{array}{l}\text { Disease } \\
\text { duration } \\
\text { (years) }\end{array}$ & $\begin{array}{c}\text { Lung } \\
\text { involvement }\end{array}$ & $\begin{array}{l}\text { Skin sclerosis } \\
\text { degree }\end{array}$ \\
\hline $\mathrm{P} 1$ & 64 & $\mathrm{~F}$ & ANA & 1 & ILD & Severe \\
\hline $\mathrm{P} 2$ & 62 & $\mathrm{~F}$ & ANA & 1 & ILD & Severe \\
\hline P3 & 65 & $\mathrm{~F}$ & ANA & 1 & - & Mild \\
\hline $\mathrm{P} 4$ & 32 & $\mathrm{~F}$ & Scl70 & 2 & - & Severe \\
\hline P5 & 58 & $\mathrm{~F}$ & Scl70 & 1 & ILD & Severe \\
\hline P6 & 63 & $\mathrm{~F}$ & Scl70 & 4 & - & Mild \\
\hline $\mathrm{P} 7$ & 30 & M & Scl70 & 1 & ILD & Mild \\
\hline P8 & 63 & M & ANA & 2 & ILD & Mild \\
\hline H1 & 62 & $\mathrm{~F}$ & - & - & - & - \\
\hline $\mathrm{H} 2$ & 64 & $\mathrm{~F}$ & - & - & - & - \\
\hline $\mathrm{H} 3$ & 65 & $\mathrm{~F}$ & - & - & - & - \\
\hline $\mathrm{H} 4$ & 59 & $\mathrm{~F}$ & - & - & - & - \\
\hline
\end{tabular}

P, patients; H, healthy controls; ANA, antinuclear antibody; Scl-70, anti-topoisomerase I antibody;

ILD, interstitial lung disease.

2 
Table 2 (on next page)

Table 2. IGH sequence statistics.

Table 2. IGH sequence statistics. 
3 Table 2. IGH sequence statistics.

\begin{tabular}{|c|c|c|c|c|c|c|c|c|}
\hline Sample ID & $\begin{array}{l}\text { Clean } \\
\text { fragments }\end{array}$ & $\begin{array}{l}\text { Aligned } \\
\text { fragments }\end{array}$ & $\begin{array}{l}\text { Alignment } \\
\text { rate }(\%)\end{array}$ & $\begin{array}{l}\text { Fragments } \\
\text { used }\end{array}$ & $\begin{array}{l}\text { Clonotype } \\
\text { number }\end{array}$ & $\begin{array}{l}\text { Out of } \\
\text { frame }\end{array}$ & Reads & $\begin{array}{c}\text { Resample } \\
\text { reads }\end{array}$ \\
\hline H1 & 7199220 & 6979148 & 96.94 & 6157658 & 26609 & 9532 & 6060539 & 5591443 \\
\hline $\mathrm{H} 2$ & 7619011 & 7267210 & 95.38 & 6456957 & 17265 & 4620 & 6367390 & 5591443 \\
\hline $\mathrm{H} 3$ & 9445266 & 9130907 & 96.67 & 8303268 & 27477 & 9050 & 8174162 & 5591443 \\
\hline $\mathrm{H} 4$ & 8960188 & 8621604 & 96.22 & 7612364 & 25833 & 8323 & 7500096 & 5591443 \\
\hline $\mathrm{P} 1$ & 6218164 & 6138462 & 98.72 & 5689474 & 28377 & 9157 & 5591443 & 5591443 \\
\hline $\mathrm{P} 2$ & 7153755 & 6986351 & 97.66 & 6359872 & 27065 & 9826 & 6249078 & 5591443 \\
\hline P3 & 7128894 & 7030004 & 98.61 & 6508507 & 25453 & 8103 & 6399782 & 5591443 \\
\hline P4 & 7689752 & 7553632 & 98.23 & 6899318 & 26620 & 8669 & 6774501 & 5591443 \\
\hline P5 & 9373266 & 9271638 & 98.92 & 8514711 & 55567 & 23321 & 8364642 & 5591443 \\
\hline P6 & 9336294 & 9238211 & 98.95 & 8465770 & 46407 & 14962 & 8308364 & 5591443 \\
\hline P7 & 10881177 & 10800389 & 99.26 & 9940707 & 63290 & 26451 & 9772110 & 5591443 \\
\hline P8 & 9359034 & 9250877 & 98.84 & 8464035 & 49427 & 20366 & 8335203 & 5591443 \\
\hline
\end{tabular}

4

5

6 


\section{Table 3 (on next page)}

Table 3. IGHV-J pairing usage differences between SSc and Control groups. 
Table 3. IGHV-J pairing usage differences between SSc and Control groups.

\begin{tabular}{lccc}
\hline IGHV-J pairing & $\mathrm{SSc}(\mathrm{mean} \pm \mathrm{s} . \mathrm{d}) \%$ & Control(mean $\pm \mathrm{s} . \mathrm{d}) \%$ & $P$ \\
\hline IGHV1-18-J3 & $0.35 \pm 0.08$ & $0.19 \pm 0.09$ & 0.016 \\
GHV1-8-J2 & $0.16 \pm 0.12$ & $0.02 \pm 0.03$ & 0.028 \\
GHV1-8-J4 & $1.07 \pm 0.42$ & $0.35 \pm 0.39$ & 0.016 \\
IGHV3-53-J4 & $1.05 \pm 0.24$ & $0.59 \pm 0.13$ & 0.016 \\
IGHV3-9-J3 & $0.26 \pm 0.09$ & $0.04 \pm 0.05$ & 0.016 \\
IGHV3-9-J4 & $1.98 \pm 0.92$ & $0.62 \pm 0.84$ & 0.042 \\
IGHV3-9-J5 & $0.41 \pm 0.18$ & $0.10 \pm 0.14$ & 0.028 \\
IGHV4-61-J4 & $1.65 \pm 0.33$ & $1.18 \pm 0.19$ & 0.042 \\
IGHV2-5-J6 & $0.22 \pm 0.12$ & $0.47 \pm 0.06$ & 0.004 \\
IGHV2-70-J5 & $0.11 \pm 0.09$ & $0.27 \pm 0.11$ & 0.028 \\
IGHV3-20-J6 & $0.07 \pm 0.06$ & $0.36 \pm 0.31$ & 0.016 \\
IGHV3-23-J6 & $0.95 \pm 0.46$ & $1.78 \pm 0.33$ & 0.016 \\
IGHV3-7-J5 & $1.08 \pm 0.17$ & $1.61 \pm 0.43$ & 0.042 \\
IGHV4-4-J6 & $0.95 \pm 0.46$ & $2.13 \pm 0.58$ & 0.016 \\
\hline
\end{tabular}

1 